\title{
Presentation of aversive visual images in health communication for changing health behaviour (Protocol)
}

\author{
Hollands GJ, Cameron LD, Crockett RA, Marteau TM
}
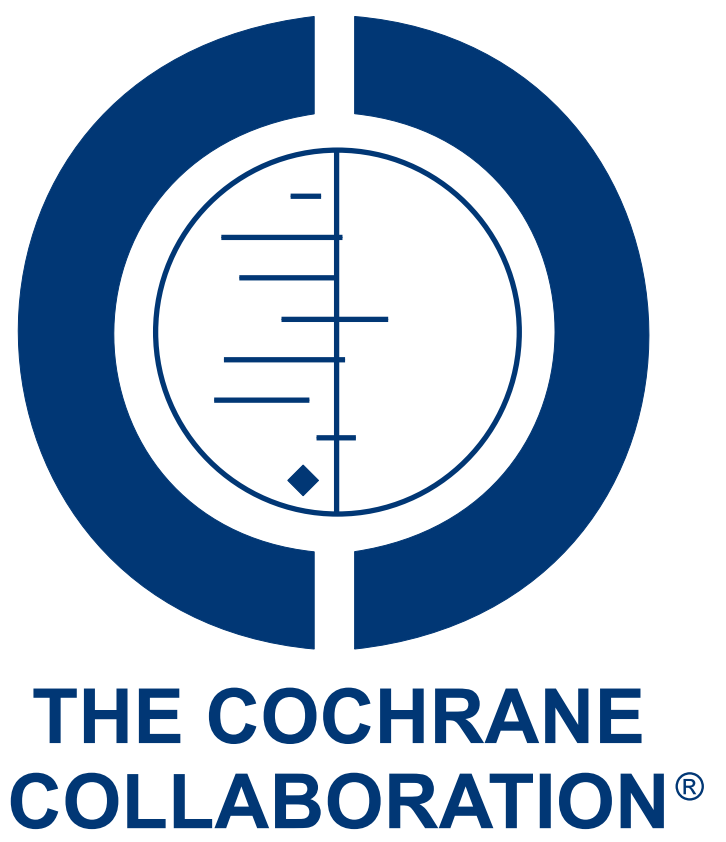

This is a reprint of a Cochrane protocol, prepared and maintained by The Cochrane Collaboration and published in The Cochrane Library 2011, Issue 4

http://www.thecochranelibrary.com

\section{WILEY}

Presentation of aversive visual images in health communication for changing health behaviour (Protocol)

Copyright $\odot 20$ II The Cochrane Collaboration. Published by John Wiley \& Sons, Ltd. 
TABLE OF CONTENTS

HEADER . . . . . . . . . . . . . . . . . . . . . . . . . . . . . . . . . . . . . . . . 1

ABSTRACT . . . . . . . . . . . . . . . . . . . . . . . . . . . . . . . . . . . . . . 1

BACKGROUND . . . . . . . . . . . . . . . . . . . . . . . . . . . . . . . . . . . . . 2

OBJECTIVES . . . . . . . . . . . . . . . . . . . . . . . . . . . . . . . . . . . . . . .

METHODS . . . . . . . . . . . . . . . . . . . . . . . . . . . . . . . . . . . . . .

ACKNOWLEDGEMENTS . . . . . . . . . . . . . . . . . . . . . . . . . . . . . . . . . . . . . . . .

REFERENCES . . . . . . . . . . . . . . . . . . . . . . . . . . . . . . . . . . . . . . 8

APPENDICES . . . . . . . . . . . . . . . . . . . . . . . . . . . . . . . . . . . . . 9.9

HISTORY . . . . . . . . . . . . . . . . . . . . . . . . . . . . . . . . . . . . . . . . 10

CONTRIBUTIONS OF AUTHORS . . . . . . . . . . . . . . . . . . . . . . . . . . . . . . . . . . . . 10

DECLARATIONS OF INTEREST . . . . . . . . . . . . . . . . . . . . . . . . . . . . . . . . . . . . .

SOURCES OF SUPPORT . . . . . . . . . . . . . . . . . . . . . . . . . . . . . . . . . . . . 11

Presentation of aversive visual images in health communication for changing health behaviour (Protocol)

Copyright $\odot 201$ I The Cochrane Collaboration. Published by John Wiley \& Sons, Ltd. 


\title{
[Intervention Protocol] \\ Presentation of aversive visual images in health communication for changing health behaviour
}

\author{
Gareth J Hollands ${ }^{1}$, Linda D Cameron ${ }^{2}$, Rachel A Crockett ${ }^{1}$, Theresa M Marteau ${ }^{1}$ \\ ${ }^{1}$ Health Psychology Section, King's College London, London, UK. ${ }^{2}$ School of Social Sciences, Humanities and Arts, University of \\ California, Merced, Merced, CA, USA \\ Contact address: Gareth J Hollands, Health Psychology Section, King’s College London, 5th Floor, Bermondsey Wing, Guy's Campus, \\ London, SE1 9RT, UK. gareth.hollands@kcl.ac.uk.
}

Editorial group: Cochrane Consumers and Communication Group.

Publication status and date: New, published in Issue 4, 2011.

Citation: Hollands GJ, Cameron LD, Crockett RA, Marteau TM. Presentation of aversive visual images in health communication for changing health behaviour. Cochrane Database of Systematic Reviews 2011, Issue 4. Art. No.: CD009086. DOI: 10.1002/14651858.CD009086.

Copyright (C) 2011 The Cochrane Collaboration. Published by John Wiley \& Sons, Ltd.

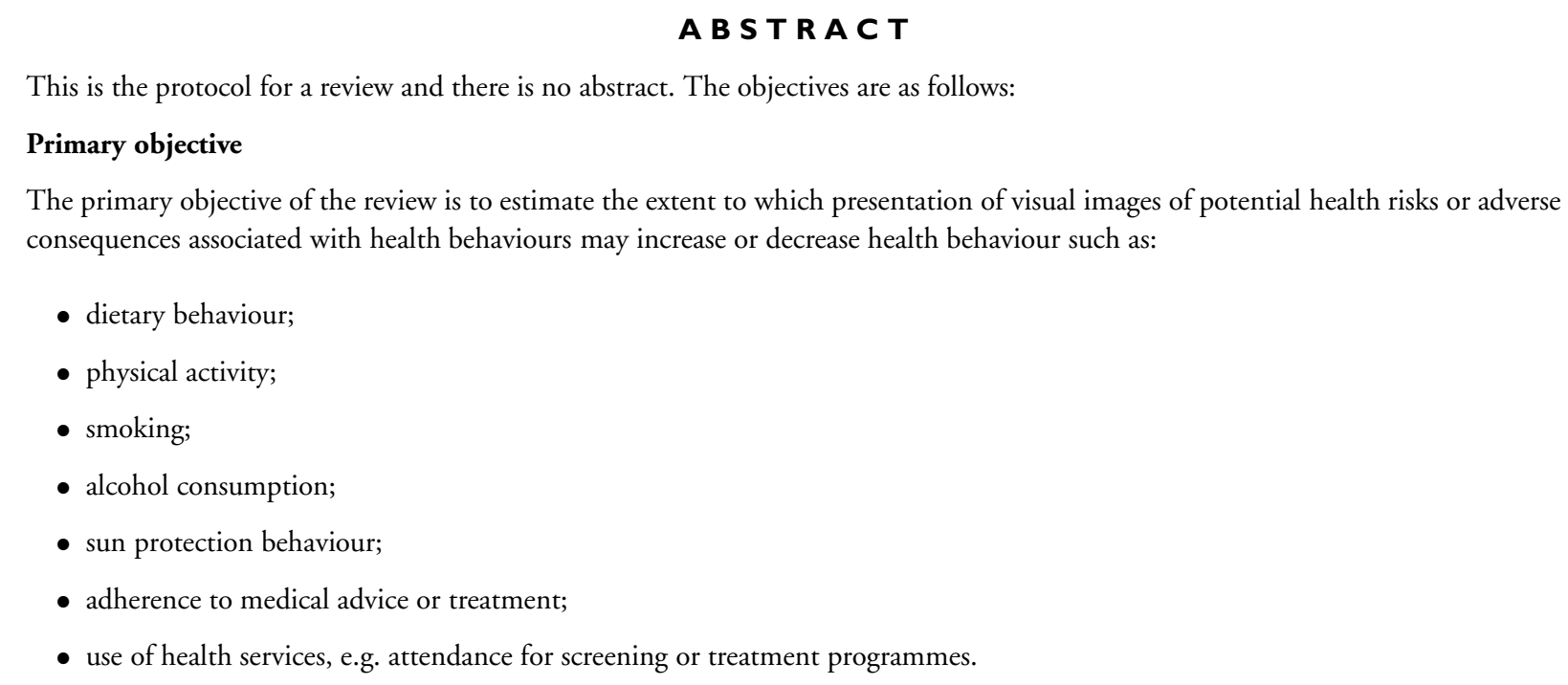

This will be considered in comparison to the impact of communicating information in a way that does not involve showing the individual such images (such as through purely written or oral communications).

\section{Secondary objective}

A secondary objective is to determine the impact of these interventions on the following outcomes (or their moderating role in behaviour change):

- intention or motivation to change health behaviour;

- knowledge and understanding of the disease, condition, treatment or risk;

- attitudes toward the disease, condition, treatment or health care;

Presentation of aversive visual images in health communication for changing health behaviour (Protocol)

Copyright $\odot 201$ I The Cochrane Collaboration. Published by John Wiley \& Sons, Ltd. 
- perceived severity of the disease or condition;

- perceived susceptibility to the disease or condition;

- self-efficacy (confidence in the ability to change behaviour);

- response efficacy (perceived effectiveness of behaviour change with regard to health);

- emotional response e.g. fear or disgust

- psychological health of the patient, including anxiety, depression, mood, wellbeing;

- acceptability of the intervention.

\section{B A C K G R O U N D}

Noncommunicable conditions, principally cardiovascular diseases, diabetes, cancers, and chronic respiratory diseases, accounted for an estimated 35 million deaths in 2005 (60\% of all deaths globally), with approximately 16 million of these deaths being in people under 70 years of age (WHO 2008). These conditions are strongly related to patterns of behaviour that may be modifiable. Achieving health behaviour change is both important, and difficult. To this end, there is an ongoing interest in developing interventions which encourage behaviour change.

\section{Description of the intervention}

One intervention strategy that has received attention in recent years, particularly in preventive medicine and population healthcare settings, is the use of aversive visual images that graphically illustrate the potential health risks or adverse consequences associated with health behaviour. A key example, applied internationally, is the use of graphic picture warnings on cigarette packaging designed to stop people smoking. Health promotion campaigns regularly make use of aversive images, such as a recent mass media campaign in the UK that showed the damaging unseen effects of alcohol on internal organs (Department of Health 2010).

As with other social interactions, health communications, even those centred around the communication of images, rarely operate on a purely visual level, and may consist of both visual and verbal stimuli. Thus it may not always make sense to draw a dichotomy between what are often complementary sources of information, and in some contexts, the effect of the addition of images to a given communication is what will be assessed by this review. Even if communication is predominantly visual in nature, its meaning may not be transparent, and limited written information may be added (as is the case with graphic picture warnings on cigarette packaging).

\section{How the intervention might work}

The development and assessment of health behaviour change interventions has traditionally been rooted in models of rational decision-making which view our actions as reasoned and intentional, requiring conscious control. Numerous models of healthrelated decisions and behaviour have been developed that emphasise the processes of rational cognitive appraisal of threat and response (Ajzen 1991; Rogers 1983; Schwarzer 2001). It follows from this approach that providing information that allows the recipient to reflect on the appropriate healthy choice may be sufficient to effect change. However, in many behavioural contexts this has not been borne out. As such, more recently, researchers have adopted a more comprehensive approach to understanding behaviour change, with the development of dual-system models of behaviour (e.g. Gawronski 2006; Strack 2004). Such models propose two systems of information processing, consisting of the processes of reflective, reasoned processing mentioned earlier, together with the processes of learned associations that are outside of conscious awareness and give rise to behavioural impulses. The latter are defined by their relation to specific cues in the environment, and having a strong emotional component, as well as providing a behavioural urge to act or approach a stimulus.

In questioning the concept of the rational decision-maker operating on a largely individual basis, we must also consider other situational and environmental factors which can limit or direct individuals' behaviour beyond their immediate conscious control. For example, socio-economic status has been shown to be a significant determinant of health behaviour, such as smoking (Eek 2010). We introduce the potential roles of such non-conscious processing and environmental influences in behaviour change to give a sense of the wider theoretical context that may explain the effects of behavioural interventions. Whilst we will not systematically assess these factors in the current review, we will consider them as effect modifiers when contextualising the evidence.

When mainly focusing on conscious, rational processing at the level of the individual, the mechanisms underlying behaviour change remain complex. However, they can be conceptualised as 
dependent on two sets of processes: motivational (forming an intention to undertake a given behaviour) and volitional (actually performing the behaviour) (Schwarzer 2001). Forming an intention is a moderately strong predictor of behaviour, but is not an absolute predictor (Webb 2006). Incorporating aversive visual images as a component of an intervention may enhance the communication of health risk and health-related information and thus alter an individual's motivational processing concerning a recommended action. This may be effective because, as with the processing of emotional information, visual information and its meaning is thought to be readily assimilated, whilst abstract language requires more analysis (Dansereau 2009). In cognition research, the 'picture superiority effect' is a well-documented finding: stimuli presented as images are better remembered on tests of recall or discrimination than equivalent stimuli presented as words (Hockley 2008; Mintzer 1999). Research on the use of visual images for enhancing health communication is limited, but has shown that pictures linked to written or spoken text can significantly increase attention to, recall, and comprehension of health information (Brotherstone 2006; Houts 2006).

\section{Why it is important to do this review}

Using aversive visual images to communicate health information may hold intuitive appeal and be considered more potent and influential than non-visual means of communication. This is ever more so, within a context of the increasing application of visual imagery in medical science and social communication, and by extension, where these spheres overlap, in clinical and preventive healthcare settings. Presently, aversive imagery is included in warning messages for smoking cessation internationally, including in the UK, Canada, Australia, Egypt and Brazil. Ongoing empirical assessment of the actual efficacy of such interventions in changing behaviour is therefore of great interest, as is evidence which may suggest the value of similar approaches in different clinical and behavioural contexts. Whilst interventions that focus on providing information to a relatively passive recipient have typically been associated with small effects on behaviour change (Albarracin 2005; Stadler 2010), the opportunity for widespread application means that there is inherent potential for aggregate impact as a population-based strategy.

This review will gather a coherent body of experimental evidence to examine systematically the behavioural impact of presenting aversive visual images. Previous reviews of a range of aversive communications (e.g. Witte 2000; de Hoog 2007) whilst comprehensive in scope, have not utilised methods able to adequately determine this. Furthermore, we will examine the cognitive and emotional mediators and moderators of any behavioural change, and make recommendations for future research and application. We are confident that there are now sufficient studies, including several in press of which we are aware, to make a review of the area viable.

\section{O B J E C T I V E S}

\section{Primary objective}

The primary objective of the review is to estimate the extent to which presentation of visual images of potential health risks or adverse consequences associated with health behaviours may increase or decrease health behaviour such as:

- dietary behaviour;

- physical activity;

- smoking;

- alcohol consumption;

- sun protection behaviour;

- adherence to medical advice or treatment;

- use of health services, e.g. attendance for screening or treatment programmes.

This will be considered in comparison to the impact of communicating information in a way that does not involve showing the individual such images (such as through purely written or oral communications).

\section{Secondary objective}

A secondary objective is to determine the impact of these interventions on the following outcomes (or their moderating role in behaviour change):

- intention or motivation to change health behaviour;

- knowledge and understanding of the disease, condition, treatment or risk;

- attitudes toward the disease, condition, treatment or health care;

- perceived severity of the disease or condition;

- perceived susceptibility to the disease or condition;

- self-efficacy (confidence in the ability to change behaviour);

- response efficacy (perceived effectiveness of behaviour change with regard to health);

- emotional response e.g. fear or disgust

- psychological health of the patient, including anxiety, depression, mood, wellbeing;

- acceptability of the intervention.

METHODS

Criteria for considering studies for this review

Types of studies 
Randomised controlled trials (RCTs), cluster randomised controlled trials (e.g. by community or region) or quasi-RCTs (those without truly random assignment). We will also include controlled before and after (CBA) and interrupted time series (ITS) studies, but limited to those which meet the criteria specified by the Cochrane Effective Practice and Organisation of Care Review Group (EPOC) (Ryan 2009). For CBA designs, this means that the timing of data collection for the control and intervention groups should be the same and that both sites should be comparable on key characteristics i.e. with respect to demographics and intervention context. For ITS designs, this means having a clearly defined point in time when the intervention occurred and at least three data points before and three after the intervention. The inclusion of non-RCT designs is necessitated by the difficulty of applying true randomised trials to studies of population-level interventions. For example, when thinking about evaluating mass media interventions, that may comprise billboard or television advertising, it is not feasible to randomise each individual and ensure that they are only exposed to a specific intervention. As such, we expect a small number of eligible RCTs.

\section{Types of participants}

Adult (18+ years old) individuals to whom the aversive visual image is directed. Participants' health status will not determine eligibility but we will extract this information and report it in the description of included studies.

\section{Types of interventions}

Interventions consist of the presentation of aversive visual images of potential health risks or adverse consequences associated with a health behaviour, as the sole or principal component.

Not all possible types of visual image are eligible. Images in this context are defined as photographs or pictures, either still or moving, which are representational (i.e. recognisable from the real world) rather than abstract in form. They are concrete representations of the body's structure, anatomy or pathology and translate its visual properties directly. This means that both text and graphs, which are ways of representing equivalent information, and can be visualised, are regarded as qualitatively different and beyond the remit of this review. Graphs may represent an object at one level, but do not directly translate the physical properties of that object as if it were being visualised by the eye.

We define 'aversive' as intending to evoke a negative emotional response, such as fear or disgust. Where this intention is not clearly addressed by study authors, and the aversive (or otherwise) nature of the image used is ambiguous, we will discuss this and form a consensus as to whether an image is aversive or not.

We will include interventions irrespective of clinical context and means of delivery. Therefore interventions applied within primary care, inpatient or community contexts will all be eligible for inclusion, as will studies that involve delivery by individuals or through mass-media methods (e.g. advertisements, product packaging). Included images will not feature images of the individual being targeted by the intervention and so we will exclude interventions which use medical imaging techniques to image the individual in question. Such interventions are covered in another Cochrane review (Hollands 2010).

We will include complex multiple-component interventions in which communication of images is one of an array of interventions, provided we can ascertain an effect-size for that component.

Acceptable comparison groups are those that either:

1. provide risk/health information communicated without the use of images; or

2. provide no risk/health information.

\section{Types of outcome measures}

Included studies must report a behavioural outcome. All outcomes will be measured either by means of a behavioural endpoint using an electronic or chemical measure, or through a self-report or questionnaire measure.

\section{Primary outcomes}

\section{Health behaviour}

Engagement in health-related behaviours that have the potential to modify the health risk of the participant, such as, but not limited to:

- diet;

- physical activity;

- smoking;

- alcohol consumption;

- sun protection behaviours;

- adherence to medical advice or treatment;

- use of health services (e.g. attendance for screening, treatment programmes).

\section{Secondary outcomes}

Cognitive and emotional mediators and moderators, and additional impacts of the intervention, such as:

\section{Health behaviour}

- intention or motivation to change health behaviour

- attitudes toward the disease, condition, treatment or health care

- perceived severity and level of risk of the disease or condition

- perceived susceptibility to the disease or condition

- self-efficacy regarding behaviour change 
- response efficacy (perceived effectiveness of behaviour change with regard to health)

- emotional response e.g. fear or disgust

\section{Knowledge and understanding}

- knowledge and understanding of the disease, condition, treatment or risk

\section{Health status and wellbeing}

- psychological health of the patient, including anxiety, depression, mood, wellbeing

\section{Evaluation of care}

- acceptability of the intervention

\section{Treatment outcomes}

- adverse events reported (e.g. clinical levels of depression or anxiety)

\section{Service delivery level}

- costs associated with featured interventions

\section{Search methods for identification of studies}

\section{Electronic searches}

We will search the following databases:

- Cochrane Central Register of Controlled Trials

(CENTRAL, The Cochrane Library) (latest issue)

- MEDLINE (OVID SP) (1950 to present)

- EMBASE (OVID SP) (1980 to present)

- CINAHL (EBSCO Host) (1982 to present)

- PsycINFO (OVID SP) (1806 to present)

The search strategies will comprise searches both for keywords and medical subject headings under existing database organisational schemes. We present the strategy for MEDLINE (Ovid SP) in Appendix 1.

We will search the ProQuest Dissertations and Theses database for grey literature.

We will search databases in the metaRegister of Randomised Controlled Trials to identify ongoing studies. If applicable, we will present relevant ongoing studies in a table in the review.

\section{Searching other resources}

We will attempt to contact authors of all included studies (along with other key researchers in the field) to identify other studies, and to ascertain further details of methodology and data of included studies.

We will search reference lists of relevant studies and systematic reviews. We will not handsearch journals.

\section{Data collection and analysis}

\section{Selection of studies}

Two review authors will independently pre-screen all search results (titles and abstracts) for possible inclusion, and those selected by either or both authors will be subject to full-text assessment. Two review authors will independently assess the full-text articles for inclusion. Any discrepancies will be resolved by consensus, overseen by a third review author acting as arbiter, with approval by one review author and the arbiter being sufficient. We will list those studies excluded after full-text assessment in the table 'Characteristics of Excluded Studies', giving reasons for exclusion.

\section{Data extraction and management}

We will develop a data extraction form based on the Cochrane Consumers and Communication Review Group's template, and pilot and amend it as necessary. We will extract the following main sets of data from each included study:

- lead author;

- date;

- study participant inclusion criteria;

- participants (participant condition(s) and demographics: race/ethnicity, gender, religion/culture, socioeconomic status, age);

- study design and timetable; randomisation; allocation concealment;

- interventions (content and format of interventions, including details of oral or written information provided; nature of images shown to participants; intervention setting and delivery provider; delivery of any co-interventions, theoretical basis of intervention if stated);

- numbers of participants in each trial arm;

- outcome measures; time(s) at which outcomes assessed;

- results;

- confounders;

- analysis methods;

- additional comments.

At least two review authors will extract data independently to the data extraction form. The forms will then be checked by a third review author and any errors or inconsistencies resolved. The first 
review author will enter the data into RevMan, with another review author checking the accuracy of the entered data.

\section{Assessment of risk of bias in included studies}

For RCTs, cluster-randomised trials and CBA designs, we will assess and report on the risk of bias of included studies by outcome, in accordance with the guidelines in the Cochrane Handbook for Systematic Reviews of Interventions (Higgins 2009), which recommends the explicit reporting of the following individual domains:

- sequence generation;

- allocation concealment;

- blinding of study personnel, data analysts and outcome assessors (assessed for each main outcome or class of outcome);

- incomplete outcome data (assessed for each main outcome or class of outcome);

- selective outcome reporting;

- other sources of bias (comparability of group characteristics, suitability of control group in terms of contact/intervention received).

We will also examine and report the following:

- validation and reliability of outcome measures;

- whether the study obtained ethics committee approval and ensured informed consent for participation;

- consistency in intervention delivery (i.e. was the information standardised/scripted and was fidelity to protocol monitored).

In line with the Cochrane Handbook (Higgins 2009) and additional EPOC guidance (EPOC 2009) specific considerations will be applied to ITS designs. For these studies, we will assess risk of bias associated with the following seven domains: the intervention being independent of other changes; the shape of the intervention effect being pre-specified; the intervention being unlikely to affect data collection; blinding of outcome assessors to intervention allocation; incomplete outcome data being adequately addressed; selective reporting of outcomes; and other sources of bias.

Two review authors will independently assess the risk of bias in included studies, with any disagreements resolved by discussion and consensus, and with a third review author acting as arbiter. We will use the aforementioned criteria to judge whether a study has a low, high or unclear risk of bias for each criterion. We will present our assessment in risk of bias tables for each included study including a descriptive summary of our decision.

We will use the GRADE system to assess the quality of the evidence for each individual outcome across studies and to produce a Summary of Findings table (Higgins 2009).

We will contact study authors for additional information about their study methods, as necessary.

\section{Measures of treatment effect}

We will present studies' major characteristics and results, presenting each primary outcome (health behaviour) separately. This will include baseline and follow-up summary statistics and effect size estimates and statistical significance. For ITS designs, summary statistics will be given, and we intend to report both the change in level of the outcome at the first time point following the introduction of the intervention, and the difference in the slopes for the levels of outcomes pre and post-intervention (i.e. post-intervention slope minus pre-intervention slope). These estimates are calculated from regression models adjusting for autocorrelation. For continuous outcomes, measures will be standardised to have common effect sizes, defined as the standardised mean difference (SMD). The effect measure for dichotomous outcomes will be the risk ratio (RR). Where varying length follow-up data are reported, we will report results taken from the furthest points in time relative to the intervention.

\section{Unit of analysis issues}

The results of cluster-randomised trials, quasi-randomised trials and CBA designs in which individual participants are allocated in clusters to intervention groups, should account for correlation of measurements within clusters. If an analysis is reported that accounts for this element of the design, the effect will be estimated on this basis. If this is not reported or the information is not available from authors, then an 'approximately correct' analysis will be carried out according to current guidelines (Higgins 2009). We will impute estimates of the intra-cluster correlation (ICC), using estimates derived from similar studies (Ukoumunne 1999), other studies included in the review (if possible), or by using general recommendations from empirical research (Campbell 2000). We will report details of any such procedures. If this is not possible, we will give the effect estimate as presented but report the unit of analysis error.

\section{Dealing with missing data}

We will conduct intention-to-treat (ITT) analyses accounting for missing data where possible, either using ITT data as provided by study authors or, for dichotomous outcomes when ITT data are not provided, taking a conservative approach, with the assumption that drop-out implies the incidence of risk-increasing behaviour. For example, for smoking cessation outcomes we will follow the principle that missing data are usually regarded as 'continued smoking. When ITT is not possible, either due to missing data, or when the outcomes are reported as continuous data, due to the problematic nature of imputation we will analyse outcomes as reported. We will also report on levels of drop out in the intervention and comparison groups as an indicator of 'acceptability' of the intervention, and the likelihood of bias due to attrition. When summary statistics are unavailable from reports 
and through contact with authors, we will attempt to derive these from the manipulation of reported data. Any imputation will be explicitly reported.

\section{Assessment of heterogeneity}

We will examine heterogeneity by inspecting overlapping confidence intervals (CIs) and further quantify this using the $\mathrm{I}^{2}$ statistic (which describes the percentage of the variability in effect estimates that is due to heterogeneity rather than to sampling error). A value greater than $50 \%$ will be considered to represent substantial inconsistency in findings (Higgins 2009). We intend to assess any contribution from outliers without overlapping CIs.

\section{Assessment of reporting biases}

We will assess for small study effects using funnel plots to informally examine any relationship between study size and effect size (Sutton 2000).

\section{Data synthesis}

We will describe and summarise the findings of the included studies. If studies that assess a given behaviour are sufficiently similar in terms of population, inclusion criteria, interventions and/or outcomes (including the time(s) at which these are assessed), we will consider pooling the data relating to individual behavioural outcomes using meta-analytic methods. We will only conduct metaanalyses on results of RCTs and cluster RCTs deemed to be at low risk of bias. Studies will be judged as being at a low risk of bias if they meet all of the key criteria previously outlined (i.e. relating to sequence generation, allocation concealment, blinding of study personnel, incomplete outcome data, selective outcome reporting and comparability and suitability of control group). Other studies, if included in the review, will be tabulated in a form additional to the meta-analyses, the findings described, summarised and included in the Discussion.

In studies that report multiple time points for outcome measurement, we will report the longest follow-up time. If multiple outcomes are reported for a given behaviour, we will select the primary outcome that is indicated in the trial report. If this is not indicated, we will select that which we agree to have the greatest clinical importance.

We will use a random-effects model for pooled analysis to obtain a pooled effect size with a $95 \%$ confidence interval (CI), reflecting the heterogeneity likely to arise from the use of different settings, participant groups, disease areas, interventions and measures across the studies. The random-effects method and the fixed-effect method will give identical results when there is no heterogeneity among the studies. Where there is heterogeneity, CIs for the average intervention effect will be wider if the random-effects method is used rather than the fixed-effect method, and corresponding claims of statistical significance will be more conservative.
We will use Mantel-Haenszel meta-analytic methods (Mantel 1959) to analyse dichotomous outcomes. These are the default methods in the Review Manager software to be used in the analysis (Cochrane Collaboration 2008), and are considered the most appropriate when data are sparse, either in terms of event rates being low or study size being small (Higgins 2009). In such cases, the estimates of the standard errors of the effect that are used in inverse variance methods may be poor. We will use inverse variance methods to analyse continuous outcomes.

\section{Subgroup analysis and investigation of heterogeneity}

The included studies are likely to be heterogeneous in terms of the health risks being communicated and the behaviours that could reduce these risks. We also expect that there will be a range of settings in which these interventions will be used. We will consider this heterogeneity when evaluating the review's results, but do not anticipate undertaking a formal subgroup analysis because it is unlikely that enough readily-grouped studies will be found, and because of the lack of a clear clinical or theoretical imperative for such analysis. If subgroups which allow for the investigation of interesting or important questions with a strong rationale become clearly apparent during the review process, well-specified subgroup analysis may be considered in future updates of the review.

\section{Sensitivity analysis}

We will undertake sensitivity analysis to examine the impact of missing data, comparing results following ITT analysis to data actually found, and reporting if there are any significant differences. For meta-analysis of effects on primary outcomes we will use sensitivity analysis to examine how the pooled intervention effect is affected by the inclusion of trials determined to be at unclear or high risk of bias (as defined in Data synthesis). Finally, we will undertake an analysis to examine whether the intervention effect is significantly affected by our imputation of assumed ICCs.

\section{Consumer input}

For systematic reviews to be relevant to policy and practice, potential users of the review should be involved in the process. This involvement can help ensure that questions pertinent to these groups are addressed and that a range of outcomes are included. Furthermore, this may encourage review authors to present their findings in an accessible way. This protocol and the subsequent review will be peer reviewed by at least one consumer, as part of the Cochrane Consumer and Communication Review Group's standard editorial process. We will also seek additional feedback from members of the Cochrane Consumer Network at draft review stage.

\section{ACK NOW LEDGEMENTS}


The authors thank all those who have commented on the protocol throughout its development. In particular we would like to thank Simon Lewin (Contact Editor), Megan Prictor and Jessica Thomas (Managing Editors), Sophie Hill (Co-ordinating Editor) and John Kis-Rigo (Trials Search Co-ordinator). We also greatly appreciate the input of the peer reviewers of the Cochrane Consumers and Communication Review Group.

\section{R E F E R E N C E S}

\section{Additional references}

\section{Ajzen 1991}

Ajzen I. The theory of planned behaviour. Organisational Behavior and Human Decision Processes 1991;50:179-211.

\section{Albarracin 2005}

Albarracin D, Gillette JC, Earl AN, Glasman LR, Durantini $\mathrm{MR}, \mathrm{Ho} \mathrm{M}-\mathrm{H}$. A test of major assumptions about behavior change: A comprehensive look at the effects of passive and active HIV-prevention interventions since the beginning of the epidemic. Psychological Bulletin 2005;131:856-97.

Brotherstone 2006

Brotherstone H, Miles A, Robb KA, Atkin W, Wardle J. The impact of illustrations on public understanding of the aim of cancer screening. Patient Education and Counseling 2006;63:328-35.

\section{Campbell 2000}

Campbell M, Grimshaw J, Steen N. Sample size calculations for cluster randomised trials. Changing Professional Practice in Europe Group (EU BIOMED II Concerted Action). Journal of Health Services Research and Policy 2000;5:12-16.

\section{Cochrane Collaboration 2008}

Cochrane Collaboration. Review Manager (RevMan). 5.0. Copenhagen: The Nordic Cochrane Centre, 2008.

\section{Dansereau 2009}

Dansereau DF, Simpson DD. A picture is worth a thousand words: The case for graphic representations. Professional Psychology: Research and Practice 2009;40(1):104-10.

\section{de Hoog 2007}

de Hoog N, Stroebe W, de Wit JBF. The impact of vulnerability to and severity of a health risk on processing and acceptance of fear arousing communications: A metaanalysis. Review of General Psychology 2007;11:258-85. [DOI: $10.1037 / 1089-2680.11 .3 .258$ ]

Department of Health 2010

Department of Health. New NHS Campaign

Reveals Unseen Alcohol Damage. http:// webarchive.nationalarchives.gov.uk/+/www.dh.gov.uk/en/ MediaCentre/Pressreleasesarchive/DH_111622 2010.

\section{Eek 2010}

Eek F, Östergren P, Diderichsen F, Rasmussen NK, Andersen I, Moussa K, et al.Differences in socioeconomic and gender inequalities in tobacco smoking in Denmark and Sweden; a cross sectional comparison of the equity effect of different public health policies. BMC Public Health 2010;10:9.

EPOC 2009

Cochrane Effective Practice and Organisation of Care Group. Risk of bias. EPOC resources for review authors 2009

\section{Gawronski 2006}

Gawronski B, Bodenhausen GV. Associative and propositional processes in evaluation: An integrative review of implicit and explicit attitude change. Psychological Bulletin 2006;132:692-731.

\section{Higgins 2009}

Higgins JPT, Green S (editors). Cochrane Handbook for Systematic Reviews of Interventions Version 5.0.2 [updated September 2009]. The Cochrane Collaboration, 2009.

Hockley 2008

Hockley WE. The picture superiority effect in associative recognition. Memory and Cognition 2008;36:1351-9.

\section{Hollands 2010}

Hollands GJ, Hankins M, Marteau TM. Visual feedback of individuals' medical imaging results for changing health behaviour. Cochrane Database of Systematic Reviews 2010, Issue 1. [DOI: 10.1002/14651858.CD007434.pub2]

Houts 2006

Houts PS, Doak CC, Doak LG, Loscalzo MJ. The role of pictures in improving health communication: a review of research on attention, comprehension, recall, and adherence. Patient Education and Counselling 2006;61:173-90.

\section{Mantel 1959}

Mantel N, Haenszel W. Statistical aspects of the analysis of data from retrospective studies of disease. Journal of the National Cancer Institute 1959;22:719-48.

\section{Mintzer 1999}

Mintzer MZ, Snodgrass JG. The picture superiority effect: Support for the distinctiveness model. The American Journal of Psychology 1999;112(1):113-46.

\section{Rogers 1983}

Rogers RW. Cognitive and physiological processes in fear appeals and attitude change: A revised theory of protection motivation. In: Cacioppo BL, Petty LL editor(s). Social Psychophysiology: A Source Book. London: Guildford Press, 1983:153-76. 
Ryan 2009

Ryan R, Hill S, Broclain D, Horey D, Oliver S, Prictor M. Study Design Guide. http://www.latrobe.edu.au/chcp/ cochrane/resources.html (accessed 28 October 2010). July 2009.

Schwarzer 2001

Schwarzer R. Social-cognitive factors in changing healthrelated behaviors. Current Directions in Psychological Science 2001;10(2):47-51.

Stadler 2010

Stadler G, Oettingen G, Gollwitzer PM. Intervention effects of information and self-regulation on eating fruits and vegetables over two years. Health Psychology 2010;29: 274-83.

Strack 2004

Strack F, Deutsch R. Reflective and impulsive determinants of social behaviour. Personality and Social Psychology Review 2004;8:220-47.

Sutton 2000

Sutton AJ, Duval SJ, Tweedie RL, Abrams KR, Jones DR. Empirical assessment of effect of publication bias on meta- analyses. BMJ 2000;320:1574-7.

Ukoumunne 1999

Ukoumunne OC, Gulliford MC, Chinn S, Sterne JAC, Burney PGJ. Methods for evaluating area-wide and organisation-based intervention in health and health care: a systematic review. Health Technology Assessment 1999;3(5): $1-75$.

\section{Webb 2006}

Webb TL, Sheeran P. Does changing behavioural intentions engender behavior change? A meta-analysis of the experimental evidence. Psychological Bulletin 2006;132: 249-68.

\section{WHO 2008}

World Health Organisation. 2008-2013 Action Plan for the Global Strategy for the Prevention and Control of Noncommunicable Diseases. Geneva: WHO, 2008.

\section{Witte 2000}

Witte K, Allen M. A meta-analysis of fear appeals: implications for effective public health campaigns. Health Education \& Behavior 2000;27:591-615.

* Indicates the major publication for the study

\section{A P P E N D I C ES}

\section{Appendix I. MEDLINE (Ovid SP) search strategy}

1. visual perception/

2. photography/

3. medical illustration/

4. audiovisual aids/

5. television/

6. motion pictures as topic/

7. exp video recording/

8. multimedia/

9. photic stimulation/

10. (image* or pictur* or pictorial or illustrat* or cartoon* or graphic* or photograph* or appearance* or animation* or film* or video* or $\mathrm{dvd}^{*}$ or cd-rom* or television or multimedia or multi-media or (visual adj (aid* or presentation or representation* or perception* or communication* ${ }^{*}$ or information or media or display* or stimuli))).tw.

11. or/ $1-10$

12. health education/

13. patient education as topic/

14. computer assisted instruction/

15. health promotion/

16. exp marketing/

17. communication/

18. persuasive communication/

19. information dissemination/

20. (health adj (communication* or information or message* or education or promotion)).tw.

21. (risk communication or health warning* or warning message*).tw.

Presentation of aversive visual images in health communication for changing health behaviour (Protocol)

Copyright (c) 201 I The Cochrane Collaboration. Published by John Wiley \& Sons, Ltd. 
22. aversive therapy/

23. avoidance learning/

24. ((fear or threat) adj2 (appeal* or arous*)).tw.

25. product labeling/

26. product packaging/

27. ((modif* or chang* $\left.^{*}\right)$ adj2 (behavio* or attitud*)).tw.

28. or/ $12-27$

29. 11 and 28

30. (aversi* or negative* or affective or threat* or danger* or disgust* or distaste* or disturbing or unpleasant* or unattractive or repel* or repuls* or repugnan*).tw.

31. (conditioning or emotion* ${ }^{*}$ or fear* or warn* or risk* or motivat* ${ }^{*}$ or persua*).mp.

32. 30 or 31

33. 29 and 32

34. randomized controlled trial.pt.

35. controlled clinical trial.pt.

36. clinical trial.pt.

37. evaluation studies.pt.

38. comparative study.pt.

39. random*.tw.

40. placebo*.tw.

41. trial.tw.

42. research design/

43. follow up studies/

44. prospective studies/

45. cross over studies/

46. (experiment* or intervention*).tw.

47. (pre test or pretest or post test or posttest).tw.

48. (preintervention or postintervention).tw.

49. time series.tw.

50. (cross over or crossover or factorial* or latin square).tw.

51. (assign* or allocat* or volunteer*).tw.

52. (control* or compar* or prospectiv*).tw.

53. (impact* or effect? or chang* or evaluat*).tw.

54. or/34-53

55. exp animals/ not humans.sh.

56.54 not 55

57.33 and 56

\section{H I S T O R Y}

Protocol first published: Issue 4, 2011 


\section{CONTRIBUTIONSOFAUTHORS}

Draft the protocol background and selection criteria: GJH, LDC, RAC, TMM

Draft the protocol methods section: GJH, LDC, RAC, TMM

Develop the search strategy: GJH, RAC

Updating the review: GJH

\section{DECLARATIONSOF INTEREST}

None known

\section{SOURCES OF SUPPORT}

\section{Internal sources}

- King's College London, UK.

Provides salary, office space and IT and library resources for three authors (GJH, RAC, TMM)

- University of North Carolina, USA.

Provides salary, office space and IT and library resources for one author (LDC)

\section{External sources}

- No sources of support supplied 